\title{
Online distributed motion planning for multi-vehicle systems
}

\author{
Ruben Van Parys, Goele Pipeleers \\ KU Leuven, BE-3001 Heverlee, Belgium \\ Department of Mechanical Engineering, Division PMA \\ ruben.vanparys@kuleuven.be
}

\section{Introduction}

Networked multi-vehicle systems have received increasing attention over the last decades. Interesting application areas include surveillance, search and rescue, automated highway systems and cooperative transportation. This work presents a novel strategy for finding optimal input trajectories for vehicles to steer them from an initial location towards a desired destination. In doing so, interaction constraints between the agents, such as attaining a formation, avoiding collisions with each other or meeting at a destination position must be satisfied, while every vehicle should respect its local constraints. The computations for solving the problem are distributed among the agents by using the Alternating Direction Methods of Multipliers (ADMM). The algorithm is further formulated in an online fashion in order to cope with disturbances and variations in the environment.

\section{Distributed formulation of the motion planning}

The motion planning problem (1) for multi-vehicle systems searches for each vehicle's time-dependent trajectory $\mathbf{x}_{i}(\cdot)$. Optimal trajectories are obtained by minimizing the sum of all vehicle objectives $J_{i}$ and are subject to local vehicle constraints, such as kinematic and dynamic limitations and collision avoidance constraints, described by a set $\mathscr{X}_{i}$. Each vehicle has several neighbors, denoted by the set $\mathscr{N}_{i}$ and an interaction constraint $g_{i j}$ implies a relation between the trajectory $\mathbf{x}_{i}$ and $\mathbf{x}_{j}$ of respectively an agent $i$ and its neighbor $j$.

$$
\begin{array}{cl}
\underset{\forall i: \mathbf{x}_{i}(\cdot)}{\operatorname{minimize}} & \sum_{i=1}^{N} J_{i}\left(\mathbf{x}_{i}\right) \\
\text { subject to } & \mathbf{x}_{i}(t) \in \mathscr{X}_{i} \\
& g_{i j}\left(\mathbf{x}_{i}(t), \mathbf{x}_{j}(t)\right)=0, \quad \forall j \in \mathscr{N}_{i} \\
& \forall t \in[0, T], \quad \forall i \in\{1, \ldots, N\} .
\end{array}
$$

The trajectories are parameterized as splines as they allow a representation with a limited number of variables and enable guaranteed constraint satisfaction with a finite set of constraints [1]. The resulting optimization problem is decoupled using ADMM [2], which results in an iterative procedure where each iteration an agent only solves a local motion planning problem, considering its own constraints and objective. By communicating with its nearest neighbors, it is possible to incorporate the neighbors' intentions. This way, the ADMM iterations converge towards optimal motion trajectories where both the local vehicle constraints and the global cooperative constraints are satisfied.

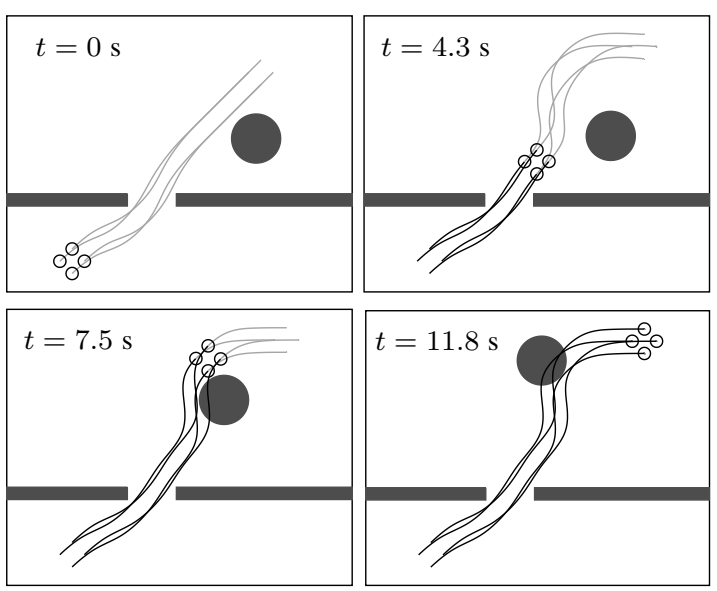

Figure 1: Motion trajectories for a formation of four holonomic vehicles in a dynamic environment. The circular obstacle starts moving at $t=4 \mathrm{~s}$ with a velocity of $(-0.15,0.15) \mathrm{m} / \mathrm{s}$. As the updates proceed, the trajectories converge towards a new optimum. The average trajectory update time equals $47.15 \mathrm{~ms}$.

\section{Extension to real-time distributed motion planning}

The algorithm is extended to a receding horizon formulation, such that the future part of the motion trajectories are reoptimized iteratively. The required update time and amount of inter-vehicle communication are reduced by performing only one ADMM iteration per trajectory update. In this way, the problem converges while the vehicles are heading towards their destination. Each update a new time horizon and a corresponding spline basis is defined. Because an ADMM iteration requires the information of trajectories of the previous iteration, their future part is first expressed in the new basis before introducing them to the next iteration.

\section{Implementation and numerical validation}

The proposed algorithm is implemented as part of a general spline-based motion planning toolbox, which facilitates the implementation and simulation of real-time motion planning problems. It is validated on numerical examples with linear as well as non-linear vehicle systems. Figure 1 considers a formation of holonomic vehicles in a dynamic environment.

References
[1] W. Van Loock, G. Pipeleers, and J. Swevers, "B-spline parameterized optimal motion trajectories for robotic systems with guaranteed constraint satisfaction," Mechanical Sciences, vol. 6, no. 2, pp. 163-171, 2015.

[2] S. Boyd, N. Parikh, E. Chu, B. Peleato, and J. Eckstein, "Distributed optimization and statistical learning via the alternating direction method of multipliers," Foundations and Trends $\AA$ in Machine Learning, vol. 3, no. 1, pp. 1-122, 2011.

Acknowledgement This work benefits from KU Leuven-BOF PFV/10/002 Centerof-Excellence Optimization in Engineering (OPTEC) and the Belgian Programme on Interuniversity Attraction Poles, initiated by the Belgian Federal Science Policy Office (DYSCO). Ruben Van Parys is a PhD fellow of the Research Foundation Flanders (FWO Vlaanderen). 